\title{
Our digital heritage as source material to end-users: Collection of and access to net publications in The National Library of Norway
}

\author{
Kjerste Rustad \\ is a Librarian, educated in 1986 from the Norwegian College of Library and Information Science, Oslo. She has been working with \\ Legal Deposit Legislation at the National Library of Norway (NLN) since 1991. Since the mid 1990s she has participated in the \\ challenges with regard to legal deposit of digital documents from the Internet. \\ Keywords: web archiving, web documents, harvesting, access, digital preservation
repository
}

Abstract The National Library of Norway (NLN) is a multimedia knowledge center that offers its users source material on any media. This paper will deal with how The National Library according to the Norwegian Legal Deposit Act collects net publications, and how these documents might be made available as source material to end-users. A web archive consisting of millions of uniform resource locators collected over several years is a challenge when it comes to access. How does the end-user get access to net publications in the internet archive for purposes of research and documentation? The paper discusses possibilities of accessing the internet archive, but also limitations set by the existing legislation.

Journal of Digital Asset Management (2006) 2, 172-177. doi:10.1057/palgrave.dam.3650032

\section{INTRODUCTION}

A researcher working on the Norwegian press history recently contacted The National Library for information. He was conducting a comparison between the news presentation in printed newspapers, broadcasting and in internet newspapers, and he wanted access to the source material. This episode might illustrate a typical end-user request. The question is, can The National Library provide this kind of information?

The National Library of Norway $(\mathrm{NLN})^{1}$ is the nation's memory, and it is a multimedia knowledge center, as stated in the Library's vision. The National Library shall preserve and make available the Norwegian cultural heritage and make records of Norwegian cultural and social life available as source material for current and future users.

So the answer to the question is YES - The The National Library of Norway,

Mo i Rana, Norway

E-mail: Kjerste.Rustad@ nb.no
National Library is expected to provide the enduser with source material on any medium. The main tool to achieve this is the Legal Deposit Act.
When the current legal deposit act came into force in 1990, Norway was among the first countries in the world that got a legal deposit legislation, which included digital documents. The Act is based on the principle that all generally available information, regardless of form or medium, must be preserved and made available as source material for purposes of research and documentation. Although the internet as we know it today did not exist in 1990, documents made generally available on the internet are subject to legal deposit according to the present Act. This is the foundation for the NLNs activity with regard to collecting net publications.

\section{COLLECTION OF NET PUBLICATIONS}

Trial harvesting of net publications has been carried out since the mid 1990s, and the first total harvesting of the entire Norwegian web space was carried out in December 2002. It was 
however in 2005 that the NLN started the regular operation of harvesting the Norwegian Internet domain.

The selection strategy is based on harvesting all generally available documents from the Norwegian web space, which is the no domain. Since the Legal Deposit Act applies to all generally available documents either produced by a Norwegian publisher or specially adapted to the Norwegian public, documents on other domains such as .org and .com might also be subject to legal deposit. This means that the NLN plans to include other domains in the longer term. Websites with robots.txt are also included. This means that the website harvester ignores the robots.txt protocol.

The robots.txt is a standard protocol that is used to ensure that search engines do not overload a website or download material which the webmaster considers irrelevant to search engines. It is common etiquette on the internet to respect robots.txt. But when it comes to the NLN and its responsibility to preserve all generally available documents on the internet, the robots.txt protocol has to be ignored to ensure that important documents actually are being preserved. Losing whole websites might be the consequence if the harvester were to follow robots.txt. This would not be in accordance with the Legal Deposit Act. It has, however, lead to discussions among net publishers, and the NLN had to argue in favor of this practice and explain that the harvesting activities are legally motivated, as distinct from any other search engine.

The NLN execute the harvesting using Heritrix $^{2}$ - a web crawler developed by the Internet Archive $^{3}$ in collaboration with, among others, the Norwegian National Library. The library's first round of harvesting net publications with Heritrix started in February 2005, and initially the ambition was to harvest the complete no domain four times a year. This was based on the calculation that each harvesting session of the Norwegian Internet domain would go on for about three weeks and collect approximately 10 million documents, that is uniform resource locators (URLs). The staff who worked on the harvesting soon experienced, however, that these calculations cracked completely. After the planned three weeks of harvesting, the web crawler had discovered more than three times as many documents as calculated, and the amount increased by the hour. By mid-July 2005, the crawler had downloaded 33.2 million URLs. It had discovered nearly 90 million URLs. The first download is still not completed, and it is expected that the number of URLs to exceed 100 million. This is more that ten times as many as expected.

The harvesting of the entire internet domain ensures the preservation of snapshots of how the Norwegian Internet domain looked at a specific moment in time. This is important source material for end-users. But it is also important to be able to preserve the entire history of one specific document. For instance, all issues of a magazine, all report in one series, and updates on a daily basis of a newspaper. The selective approach is an important supplement to the total harvesting of the all the Norwegian websites. This selective approach is step two and is our second goal regarding harvesting of net publications.

\section{INTERNATIONAL COOPERATION}

The NLN participates in various international projects for archiving the internet. The Library is part of the International Preservation Consortium (IIPC), where the Library cooperates with other national libraries and the Internet Archive in the USA. ${ }^{4}$

In addition, the Library is part of the Nordic Web Archive Project (NWA), which is the Nordic national libraries' forum for coordination of activities regarding harvesting and archiving of documents on the internet. ${ }^{5}$ Although the name of the forum might indicate that the Nordic libraries have a common web archive, this is not the case. The Nordic national libraries do separate harvests and have separate web archives. The NWA Project however, has developed a tool for accessing web archives, called the NWA Toolset.

\section{PRESERVATION OF NET PUBLICATIONS}

The National Library has a perspective of 1,000 years regarding preservation. That is, the aim is that the information on documents in any format shall be available as source material a 1,000 years from now. Whether this is a realistic 
aim, only time will tell, this is the Library's goal and the preservation activities are planned accordingly. The net publications harvested by Heritrix are stored in the National Library's Digital Long-Term Preservation Repository.

\section{ACCESS TO NET PUBLICATIONS}

How does the end-user get access to net publications in the internet archive for purposes of research and documentation? There are at least two aspects that should be taken into consideration regarding this question: metadata and legislation.

\section{Metadata}

During 2005 the NLN decided to what extent documents in the web archive should be catalogued and included in the National Bibliography. The need for bibliographic control of digital documents, how the documents should be accessed and the amount of documents on the web, are factors that have to be taken into consideration. Cataloguing the net archive will probably only be the case for less than one per cent of the documents. This means that only a tiny fraction of the documents collected from the Norwegian Internet domain might be subject to bibliographic registration.

Realizing this fact, the Library needs to have other tools for gaining access to the web archive.
The solution is this: all documents in the web archive will be fully indexed using FAST $^{6}$ indexing software. This will enable users to search the documents via free text. The documents will be accessed via the NWA Toolset. This software will provide the only way of access to more than 99 per cent of the material in the archive.

In order to illustrate how the net archive will appear to the user, the figures below demonstrate the NWA Toolset, which is the primary software to access the Norwegian Internet Archive (Figures 1-4).

\section{Legislation}

Harvesting the Norwegian Internet domain enables the NLN to preserve our digital heritage. However, the existing Norwegian legislation limits the possibility for the public to access the Internet archive.

The National Library has to consider regulations in the Legal Deposit Act, the Personal Data Act and the Copyright Act under consideration when it comes to giving access to the internet archive to end-users.

The Legal Deposit Act states that documents deposited in accordance with the Act shall be made available for purposes of research and documentation. This means that the Act gives clear restrictions when it comes to making net publications available to the end-users. The

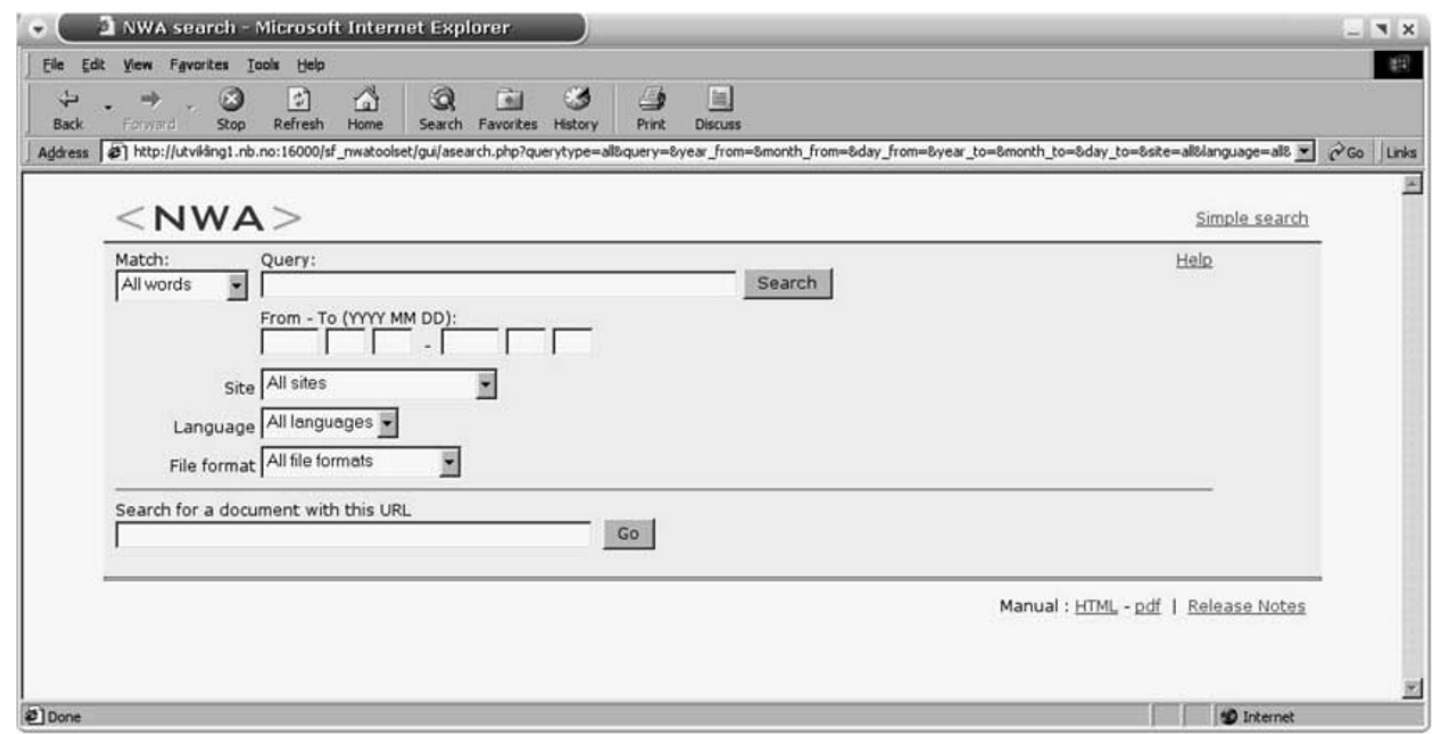

Figure 1: User interface for the advanced search in NWAToolset. The search facilities include free text search with Boolean operators and search for certain URLs. The searches might be limited by date, language and file formats, and by sites 


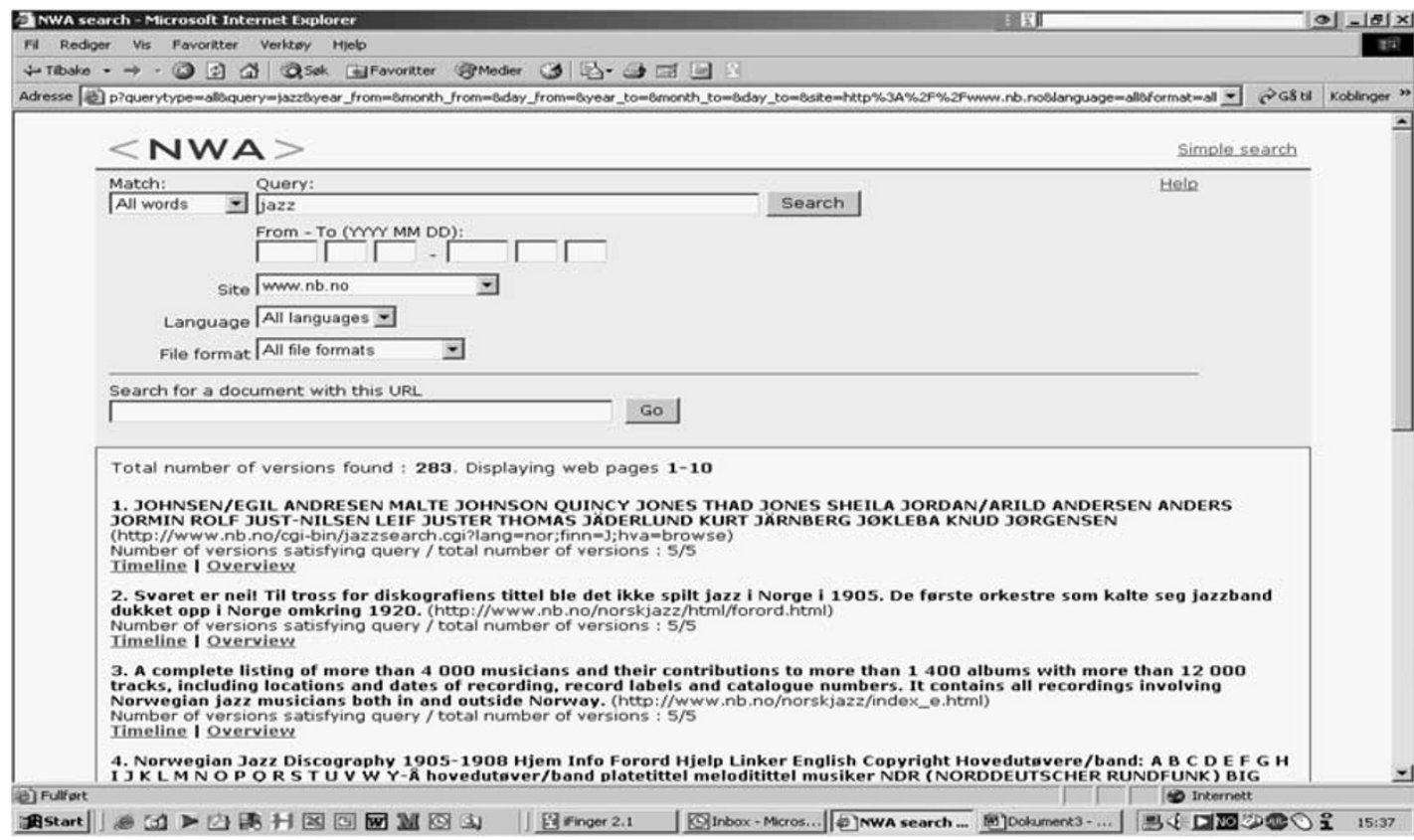

Figure 2: Example where a search for the phrase jazz, has been conducted, limited to the site of the NLN. The hit list gives two options for the presentation of each hit: timeline or overview

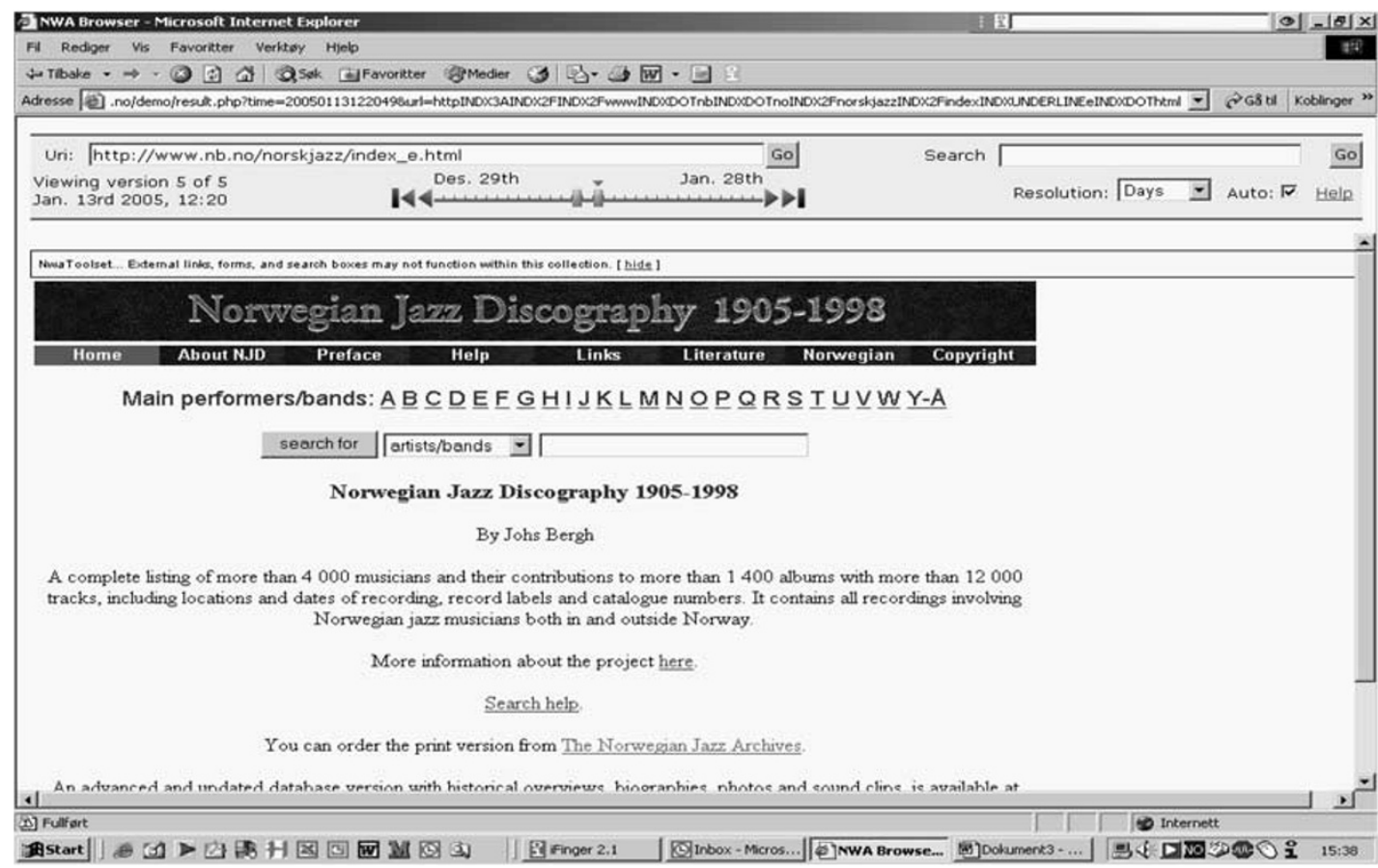

Figure 3: Presentation of the document history via a timeline. As you can see this is version 5 of five downloads of this specific document, conducted 13 January 2005 at $20 \mathrm{~min}$ past 1200 hours. The user might follow the history of the documents via the timeline. Options for resolutions of the timeline are years, months, days, hours and minutes. This gives the user the possibility of a virtual voyage in time! 


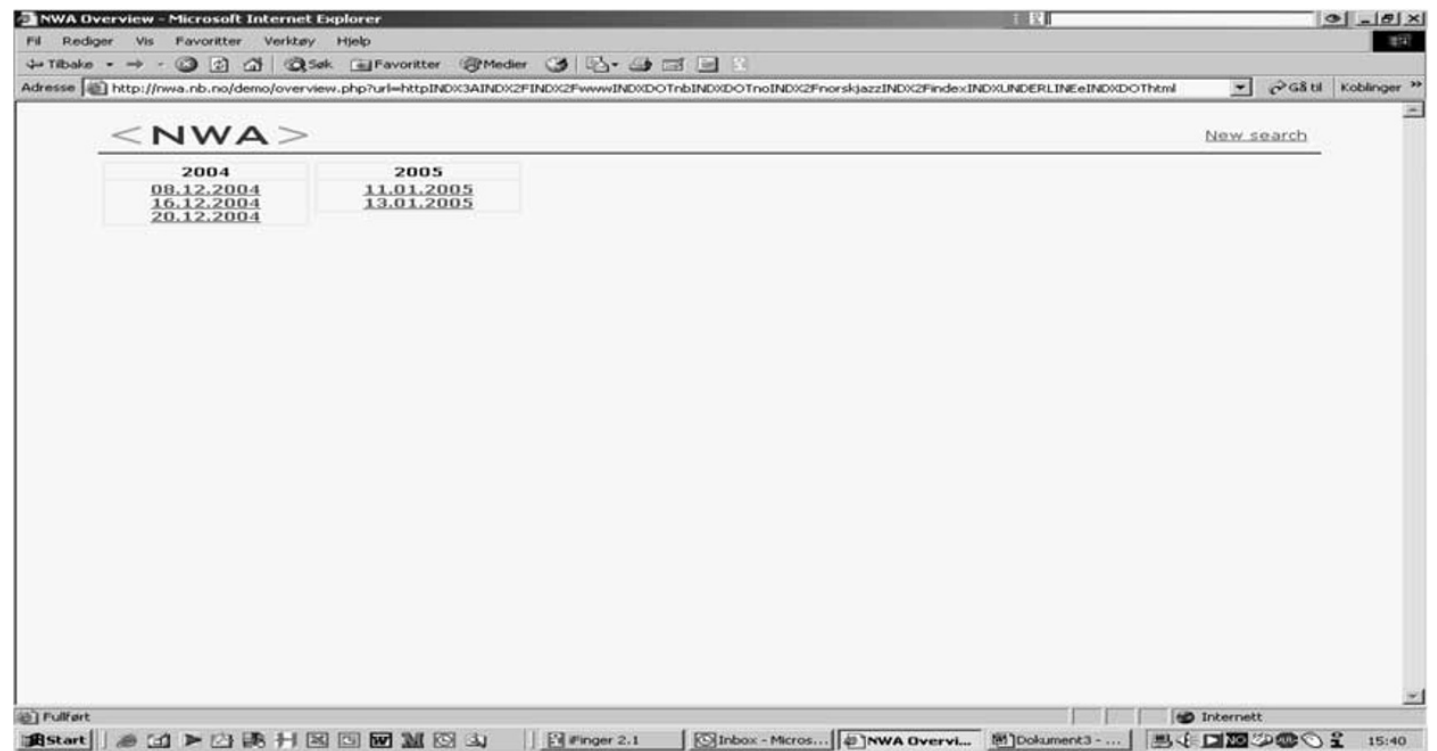

Figure 4: Presentation of the document history via an overview of the number of downloads

purpose of the use of the documents decides who can get access to the publications. Possible users who might have access to the net archive are, for instance, researchers, scholars, students, teachers and other specialized users, for example users with a specific hobby, such as genealogy, net publications, which are made available to the users, might contain both personal data and sensitive personal data. The handling of personal data is regulated in the Personal Data Act. The Act helps to ensure that personal data is processed in accordance with fundamental respect for the right to privacy, including the need to protect personal integrity and private life. The possibility of processing personal data through search facilities in the internet archive makes the information in net publications far more accessible than the traditionally published documents. This implies that the NLN has to have a license from the Norwegian Data Inspectorate to conduct the harvesting of net publications. In addition, a similar license for access is required before the Library can give access to the net publications for the end-users.

According to the Copyright Act, the NLN cannot make the deposited net publications available to the public if this access is in conflict with the copyright owners' economic and ideal rights. According to the current legislation, the NLN might give access to the net publications only on on-site computers; that is, computers within the Library's premises.

To summarize, these are the demands that have to be fulfilled according to the current legislation before The National Library might give access to end-users to the net publications in the Internet Archive:

- The use of the documents must be for purposes of research and documentation, as stated in the Legal Deposit Act.

- A general license agreement from the Norwegian Data Inspectorate according to the Personal Data Act must be signed.

- The user must visit the NLN's premises.

This leads to the recognition of the fact that the existing legislation gives the end-users less, or at least more restricted, access to digital legally deposited documents than to printed legally deposited documents.

The proposed changes in the Norwegian Copyright Act include the possibility of interlending digital documents on specific request. This means that The National Library may send a copy of a digital document upon the request from end-users situated at another library. The latter is responsible for deleting the copy after use.

Considering the fact that Norway is a sparsely populated country with large geographical distances, access to the Internet 
Archive only at The National Library premises in Oslo and Rana is not an optimal solution. We can identify four levels of access for end-users:

1. On-site access for users via The National Library's computers. According to the proposed changes in the Norwegian Copyright Act this will be permitted.

2. On-site access for users via computers in universities, colleges and public libraries. This is not in accordance with existing legislation, but would in many ways offer a good solution for access.

3. Access for users from private computers.

4. Digital documents made available via, for instance, exhibitions on The National Library's website. A license agreement will be required for this.

The access to the Internet Archive as stated in points 1 to 3 above must be limited to purposes of research and documentation. This means that open access to the public in general cannot be permitted according to existing legislation. But there are ways of giving access to net publications in the Internet Archive that might be the solution for specific parts of the archive. The NLN might sign agreements or license agreements with the copyright owners, which enable the Library to make net publications available to the public, for instance, through exhibitions on the net via the Library's home site.

The proposed changes in the Norwegian Copyright Act include the possibility of digital license agreements between libraries and copyright owner's organizations. This may give the NLN the possibility of making digital documents available to end-users, but it depends on payment.

The NLN's primary goal is to offer end-users on-site access via the Library's computers.

However, this gives the end-users a geographically limited access. The matter of future access to the web archive is now under consideration by the NLN.

\section{Which documents should the end-user have access to?}

Basically, all legally deposited net publications should be made available as source material to end-users for purposes of research and documentation. In the matter of copyright protected documentation, the access of one specific user will be limited to the part of the net archive, which is relevant for his or her needs. When it comes to documents that are not protected by the Copyright Act, such as legal statutes, administrative regulations and other decisions by public authorities, there should be no need to limit access.

\section{CONCLUSION}

Over time the Norwegian Internet Archive will consist of billions of URLs which The National Library must enable the end-user to navigate. The challenges regarding harvesting, preservation and access to web documents are huge, but the NLN believes that it has the infrastructure, the services and the organization to accomplish this.

Net publications can, from a technological viewpoint easily be made available to the public. Access is, however, limited by existing legislation, and it is vital to The National Library to ensure that all use of The Internet archive is in accordance with the Legal Deposit Act, the Copyright Act and the Personal Data Act.

The aim and responsibility as a National Library, however, is to make net publications, as records of Norwegian cultural and social life, available for end-users as source material for purposes of research and documentation.

\section{References and Notes}

1 The National Library of Norway (NLN) is the premier source of information about Norway, Norwegians and Norwegian culture, and is to be Norway's main resource for the collection, archiving and distribution of Norwegian media. Its functions include establishing, preserving and making available a wide variety of collections..

2 Heritrix (2005) Available at: http://crawler.archive. org/ (Accessed 24 August).

3 Internet Archive. (2005) Available at: http://www. archive.org/ (Accessed 24 August).

4 International Preservation Consortium. (2005) available at: http://netpreserve.org/about/index. php (Accessed August 24).

5 Nordic Web Archive Project. (2005) available at: http://nwa.nb.no/ (Accessed August 24).

6 FAST. (2005) available at: http://www.fast.no (Accessed 24 August). 